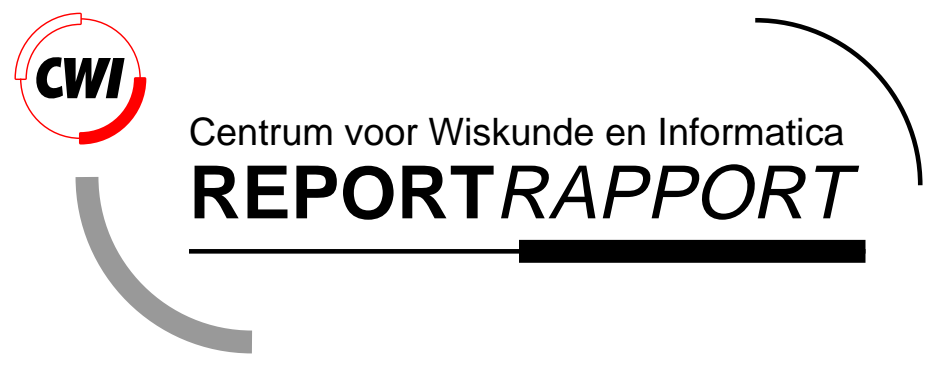

Solving Vertical Transport and Chemistry in Air Pollution Models

P.J.F. Berkvens, M.A. Botchev, J.G. Verwer, M.C. Krol, W. Peters

Modelling, Analysis and Simulation (MAS)

MAS-R0023 August 31, 2000 
Report MAS-R0023

ISSN 1386-3703

CWI

P.O. Box 94079

1090 GB Amsterdam

The Netherlands

$\mathrm{CWI}$ is the National Research Institute for Mathematics and Computer Science. CWI is part of the Stichting Mathematisch Centrum (SMC), the Dutch foundation for promotion of mathematics and computer science and their applications.

SMC is sponsored by the Netherlands Organization for Scientific Research (NWO). CWI is a member of ERCIM, the European Research Consortium for Informatics and Mathematics.

Copyright (C) Stichting Mathematisch Centrum P.O. Box 94079, 1090 GB Amsterdam (NL) Kruislaan 413, 1098 SJ Amsterdam (NL) Telephone +3120 5929333 Telefax +31205924199 


\title{
Solving Vertical Transport and Chemistry in Air Pollution Models
}

\author{
P.J.F. Berkvens, M.A. Botchev, and J.G. Verwer \\ CWI \\ P.O. Box 94079, 1090 GB Amsterdam, The Netherlands \\ [berkvens, botchev, jan.verwer]@cwi.nl \\ M.C. Krol and W. Peters \\ IMAU \\ P.O. Box 80005,3508 TA Utrecht, The Netherlands \\ [m.krol, w.peters]@phys.uu.nl
}

\begin{abstract}
For the time integration of stiff transport-chemistry problems from air pollution modelling, standard ODE solvers are not feasible due to the large number of species and the 3D nature. The popular alternative, standard operator splitting, introduces artificial transients for short-lived species. This complicates the chemistry solution, easily causing large errors for such species. In the framework of an operational global air pollution model, we focus on the problem formed by chemistry and vertical transport, which is based on diffusion, cloud-related vertical winds, and wet deposition. Its specific nature leads to full Jacobian matrices, ruling out standard implicit integration.

We compare Strang operator splitting with two alternatives: source splitting and an (unsplit) Rosenbrock method with approximate matrix factorization, all having equal computational cost. The comparison is performed with real data. All methods are applied with half-hour time steps, and give good accuracies. Rosenbrock is the most accurate, and source splitting is more accurate than Strang splitting. Splitting errors concentrate in short-lived species sensitive to solar radiation and species with strong emissions and depositions.
\end{abstract}

2000 Mathematics Subject Classification: Primary: 65M06, 65M20. Secondary: 65Y20.

1998 ACM Computing Classification System: G.1.8, J.2.

Keywords and Phrases: Numerical time integration, stiff ODEs, splitting techniques, air pollution problems.

Note: Work carried out under subtheme MAS1.1 'Atmospheric Flow and Transport Problems'.

\section{INTRODUCTION}

In global air pollution modeling the time dependent chemical composition of the atmosphere is described by a three-dimensional (3D) system of partial differential equations (PDEs),

$$
\frac{\partial c_{i}}{\partial t}=\mathcal{T}_{i} c_{i}+f_{i}\left(c_{1}, \ldots, c_{m_{s}}\right), \quad i=1, \ldots, m_{s} .
$$

The variables $c_{i}$ represent the concentrations of $m_{s}$ atmospheric trace gases, the linear term $\mathcal{T}_{i} c_{i}$ represents the transport by various atmospheric transport processes. The non-linear term $f_{i}$ describes the chemistry that occurs between between the trace gases; it may also contain emissions and depositions. In general, the transport term contains horizontal and vertical advection and diffusion. In global applications it also contains parameterizations of rapid vertical motions which occur on subgrid scales both in space and time. The chemical interaction term $f_{i}$ is generally stiff and thus requires an implicit numerical solution method. The system (1.1) is augmented with boundary conditions, which may contain emissions and depositions. Observe that without the chemical interactions $f_{i}$, the system (1.1) represents $m_{s}$ decoupled transport equations. In practical applications $m_{s}$ varies from 20 to 100 and the global atmosphere is resolved in $\mathcal{O}\left(10^{4}\right)$ to $\mathcal{O}\left(10^{6}\right)$ grid points. 
The vertical spatial discretization of the transport term on a grid of $m_{h}$ grid cells yields a system of ordinary differential equations (ODEs) which fits the form

$$
\dot{w}=F(w) \equiv T w+f(w), \quad w \in R^{N}, \quad N=m_{h} m_{s} .
$$

The linear term $T w$ is the semi-discrete vertical transport term and the nonlinear term $f(w)$ contains the chemistry. As explained above, convection is a vertical transport process that occurs on the sub-grid scale and couples the concentrations of each species in all the layers in which the convection occurs. This gives rise to a block-diagonal matrix $T$ composed of $m_{s}$ completely full blocks of dimension $m_{h}$. The immediate consequence is that the $N \times N$ Jacobian matrix $F^{\prime}(w)$ is nearly full. The Jacobian is not completely full because the Jacobian of the chemical sub-system is sparse. This sparsity, however, cannot be efficiently exploited in a direct solution of linear systems. The reason is that the sparse chemistry Jacobians of different grid cells are coupled in $F^{\prime}(w)$ by the transport matrix $T$ in such a way that the sparsity will be lost in a direct solution. Thus the straightforward use of implicit solvers is ruled out and tailored numerical integration methods are required.

An efficient and widely used method to solve such a huge system is operator splitting in which the chemistry and transport processes are advanced in time sequentially. However, splitting errors may dominate the total error for typical time step sizes used in long-term simulations. Splitting errors are caused by splitting the time step advance for a number of processes that act simultaneously, into steps for separate processes which are then carried out consecutively. For example, when the processes of vertical mixing, emissions, depositions, and chemical reactions - which act simultaneously in reality - are time-stepped one after another, this leads to splitting errors. There is one exception: if the mathematical operators linked to the processes commute, then no splitting error arises [18]. In practice, though, this never occurs.

In this paper several methods will be studied that aim at the reduction of splitting errors. Our starting point is a Strang-type operator splitting method that is widely used in air pollution modeling $[3,8,18,21,24,26,30]$. Two alternative methods will be compared to Strang splitting and their effect on the numerical solution will be investigated. The two methods are the so-called source-splitting method and the Rosenbrock method with approximate matrix factorization. The methods will be applied to a vertical column of a realistic atmospheric chemistry model [22]. Advection is left out of system (1.1), but the vertical mixing processes, emission and deposition processes, and chemistry are included.

All three methods that are studied here have in common that they avoid the use of the huge and nearly full Jacobian $F^{\prime}(w)$ in the linear system solutions. The methods will be outlined in more detail in Section 3.

In a previous study [3] it was found that splitting errors are of minor importance for longer lived species like ozone $\left(\mathrm{O}_{3}\right)$. However, for rapidly varying short-lived species (like the $\mathrm{N}_{2} \mathrm{O}_{5}$ ) standard operator splitting completely fails occasionally. These large deviations from the reference solution occurred mainly near strong emission peaks and at sunrise and sunset. The Rosenbrock method was found to provide the best solution. The model used in [3] contained only vertical diffusion as a transport mechanism. In the current study a different chemical mechanism is used and also convection type transport is included, but, different from [3], advection is left out. One aim of this paper is to reexamine the splitting errors in this different model set-up.

We address the following questions:

- How accurate are the three methods Strang splitting, source splitting, and Rosenbrock with approximate matrix factorization?

- Are these accuracies acceptable for the application field?

- How do the three methods compare among themselves?

- What are the effects of operator splitting? Particularly interesting is the question, whether for the current model, based on a vertical transport mechanism and set of chemical reactions 
Table 1: Operator notation

\begin{tabular}{lc|c}
\hline Process & \multicolumn{2}{c}{ Operator } \\
\hline Vertical diffusion & $\mathcal{V}$ & \multirow{2}{*}{$\mathcal{T}$} \\
Convection & $\mathcal{V}$ \\
Wet deposition & $\mathcal{W}$ & \\
Dry deposition & $\mathcal{D}$ \\
Gas phase chemistry & $r$ \\
Emission sources & $\varsigma$ & $f$ \\
\hline
\end{tabular}

different from [3], unacceptably large splitting errors again remain restricted to the shortlived species.

- What are the computational costs?

The contents are as follows. In Section 2 we describe the systems (1) and (2) in greater detail. Section 3 is devoted to the integration algorithms for the semi-discrete system (2). The methods are compared in a numerical experiment in Section 4 and the main conclusions are summarized in Section 5 .

\section{Model Definition}

\subsection{Meteorological model}

In this study we use a one-column version of the Chemistry Transport Model version 3 (TM3). This model was used for studies of the photochemistry of the atmosphere on numerous occasions, e.g. $[7,19]$. The same processes as included in the 3D global CTM are simulated, with the exception of advection. Processes included in the one-column model are summarized in Table 1, together with the operator notation adopted in this work.

Vertical diffusion and convection together constitute the vertical transport in the model. Diffusion [20] represents transport of trace gases by large turbulent eddies in the atmosphere due to differential heating of the surface and wind shear. Since these eddies are typically smaller than $1 \mathrm{~km}$, diffusion is a local process redistributing the air over several adjacent layers. Diffusion is strongest near the surface, and near the wind maxima in the upper troposphere (jet streams). Convection [25] represents vertical transport resulting from large-scale instability in the atmosphere. Due to condensation of water vapour, air parcels become buoyant and can quickly loft several kilometers, forming cumulus clouds. During this lofting, exchange with the air surrounding the cloud occurs. Convection can redistribute trace gases over large vertical distances. Vertical diffusion and convection are non-divergent and mass conservative, and only cause spatial coupling in the vertical direction. In the cumulus clouds, soluble trace gases can deposit on cloud droplets. When these cloud droplets rain out of the cloud, trace gases are removed from the atmosphere. This process is called wet deposition [10] and is proportional to the solubility, the scavenging efficiency of the cloud, and the trace gas concentration. Wet deposition thus removes tracer mass from the atmosphere. Likewise, dry deposition [29] removes trace gases at the surface. Soils and vegetation have the ability to absorb some trace gases. The efficiency depends on many factors such as sunlight, soil type, solubility. The gas phase photo-chemistry [13] consists of 29 trace gases, that react under the influence of temperature and sunlight. The strong dependence of some reactions on the intensity of sunlight makes this system very stiff. The tendency of photochemistry to converge to an equilibrium state is constantly perturbed by the changing photolysis rates, but also by transport, emission, and removal processes. Finally, emissions in the model are present mostly in the lowermost layer, where anthropogenic activities and natural processes occur. The only exceptions are the production of NO by lightning and airplane emissions, which occurs at higher model levels. In the rest of the paper, operator $\mathcal{T}$ represents the first three processes from Table 1 :

$$
\mathcal{T}=\mathcal{V}-\mathcal{W}
$$


The remaining processes are described by operator $f$ :

$$
f_{i}\left(c_{1}, \ldots, c_{m_{s}}\right)=r_{i}\left(c_{1}, \ldots, c_{m_{s}}\right)+\mathcal{D}_{i} c_{i}+\varsigma_{i} .
$$

The upper boundary conditions of the system are that no fluxes are allowed. At the lower boundary the fluxes are determined by the emission and deposition processes. At the surface and in the top model layers, the concentrations of some longer lived species is constrained by relaxation of the concentration to a prescribed value that represents a climatology. This is done by modifying the terms $\mathcal{D}_{i} c_{i}+\varsigma_{i}$ in $(2.2)$.

In this study a column of air over the Amazonian rainforest $\left(60^{\circ} \mathrm{W}, 0^{\circ} \mathrm{N}\right)$ is selected. This location is of particular interest because of the high solar radiation, the strong vertical diffusion and frequent occurrence of convection. Furthermore, dry deposition and natural emissions from the forest are abundant in this column, and the photochemistry is very stiff. Under these conditions splitting errors are expected to maximize. Transport is based on 6-hourly meteorological data from the European Center for Medium Range Weather Forecasting (ECMWF) model for March 1997, while initial tracer concentrations and surface emissions are taken from a full 3D TM3 simulation. Since we do not include advection in the simulations, concentrations of species with strong sources can become unrealistically high. In order to prevent this, and to retain as much resemblance to the observed atmospheric situation, a relaxation of the trace gas concentrations is applied. The concentrations are relaxed to the clean background concentrations $\boldsymbol{c}_{i}^{\text {ocean }}$ observed over the oceans surrounding the continent according to:

$$
\begin{aligned}
\mathcal{D}_{i} & :=\mathcal{D}_{i}+\tilde{\mathcal{D}}, \\
s_{i} & :=s_{i}+\tilde{s}_{i}, \quad \tilde{s}_{i}=-\tilde{\mathcal{D}} \boldsymbol{c}_{i}^{\text {ocean }},
\end{aligned}
$$

where $\tilde{\mathcal{D}}$ represents the reciprocal of the turnover time of a grid box with respect to advection. The turnover time is taken to be 10 days.

\subsection{Semi-discretization}

The vertical column is divided into $m_{h}$ cells, indexed with $k$ ( $k=1$ at the surface and $k=m_{h}$ at the top), with volumes $v_{k}$, assembled in the vector $\boldsymbol{v}$. In the present test, $\boldsymbol{v}$ does not change with time, since the advection and the related pressure changes are zero. ${ }^{1}$ The approximations of the tracer masses in the cells, which are the cell-integrals of the concentrations, are assembled in $\overrightarrow{\boldsymbol{\mu}}$ with elements $\mu_{i, k}$, where $i$ indicates the species. The average concentrations in a cell are assembled in $\overrightarrow{\boldsymbol{c}} \equiv \overrightarrow{\boldsymbol{\mu}} \oslash \overrightarrow{\boldsymbol{v}}$, where $\oslash$ means entrywise division.

The semi-discretization of the vertical-transport operator $\mathcal{T}$ yields a matrix $T$ operating on column vectors of tracer masses, thus $T \boldsymbol{\mu}_{i}$. The vertical-transport matrix can be split into a vertical-mixing matrix $V$ (corresponding to $\mathcal{V}$ ) and a convective wet deposition matrix $W$ (corresponding to $\mathcal{W}$ ), thus $T=V-W . V$ is made up of a diffusive and a convective part. For the diffusion standard central differences on a three-point stencil are used. The semi-discretization of the convection [12] results in a full matrix. This reflects the non-local nature of the vertical coupling in the parameterization of the convection [25]. The semi-discretized submodel for vertical mixing reads:

$$
\dot{\boldsymbol{\mu}}_{i}=V \boldsymbol{\mu} .
$$

Element $v_{k \ell}$ of $V, \ell \neq k$, indicates the net fraction of tracer mass $\mu_{i, \ell}$ in cell $\ell$ which moves from cell $\ell$ to cell $k$ per time unit. $V$ is conserves the tracer mass in a column.

The vertical mixing operator can be seen as a sum of an updraft operator and an operator for downdraft and subsidence. The vertical-mixing matrix $V$ can be split into an updraft part $V_{u}$ and a remaining part $V_{v}$, thus $V=V_{u}+V_{v}$, and the mass-conservation property holds for both

\footnotetext{
${ }^{1}$ We will use boldface notation for 'column' vectors of quantities varying with the computational cells, arrow notation for 'species' vectors of quantities varying with the chemical species, and a combination of boldface and arrow notation for vectors of quantities varying with both the cells and the species.
} 
of these matrices separately. $V_{u}$ is a lower-triangular matrix, which expresses the fact that the updraft only transports tracer upward. $V_{v}$ contains an upper triangular part for the downdraft and for the subsidence, and a tridiagonal part for the turbulent diffusion. The semi-discretization of the convective wet deposition yields, for each species, matrices $W_{i}$ which are closely related to $V_{u}$. They read:

$$
W_{i}=\beta_{i} E\left(V_{u}-\operatorname{diag}\left(V_{u}\right)\right),
$$

where $\beta_{i} \in[0,1]$ is the solubility of tracer $i, E=\operatorname{diag}_{k=1}^{m_{h}}\left\{\varepsilon_{k}\right\}$, with $\varepsilon_{k} \in[0,1]$, is a diagonal matrix expressing the scavenging efficiency, and $\operatorname{diag}\left(V_{u}\right)$ is the diagonal matrix containing the main diagonal of $V_{u}$.

The wet-deposition operators $\mathcal{D}_{i}$ are discretized into diagonal matrices $D_{i}$ with $d_{i, k k} \leq 0$. The diagonal elements give the cell-averaged drain rates. The volume sources $\varsigma_{i}$ are discretized into vectors $s_{i}$ whose elements contain the cell-averaged values of the sources.

With regard to the boundary conditions at the surface, after discretization the dry-depositions $\Delta_{i}$ are incorporated into the elements $d_{i, 11}$ of $D_{i}$, and the surface emissions $\sigma_{i}$ are incorporated into the element $s_{i, 1}$ of $s_{i}$, so they are attributed to the lowest cell.

Finally, the chemical reaction operator $r_{i}\left(c_{1}, \ldots, c_{m_{s}}\right)$ is semi-discretized into $r_{i}\left(c_{1}, \ldots, c_{m_{s}}\right)$ by taking cell-averaged photochemical and reaction rates operating on cell-averaged concentrations.

The above semi-discretization yields the following system of ODE's for the concentrations:

$$
\frac{\partial \boldsymbol{c}_{i}}{\partial t}=\left(T\left(\boldsymbol{c}_{i} \odot \boldsymbol{v}\right)\right) \oslash \boldsymbol{v}+D_{i} \boldsymbol{c}_{i}+\boldsymbol{s}_{i} \oslash \boldsymbol{v}+\mathbf{r}_{i}(\overrightarrow{\boldsymbol{c}}), i=1, \ldots, m_{s},
$$

where $\odot$ means elementwise multiplication. We have to transform back and forth between concentrations $\boldsymbol{c}_{i}$ and tracer masses $\boldsymbol{\mu}_{i}$, since $T$ operates on tracer masses rather than on concentrations.

In Section 3 we describe three numerical time-integration methods for solving this system of ODE's. Two of the methods use operator splitting, where for efficiency reasons the two operators $T$ and $\boldsymbol{r}_{i}$ are applied separately. This leaves us with the following choice: with which of these two operators will we combine $D$ and $s$ ? For accuracy reasons, one would choose to combine $D$ and $s$ with the operator which causes the largest splitting error if it is separated from $D$ and $s$. Because the (photo)chemical reactions $\boldsymbol{r}_{i}$ are usually more stiff than the vertical mixing $T$ for most of the species, we choose to combine both $D_{i}$ and $s_{i}$ with $\boldsymbol{r}_{i}$, and define $\boldsymbol{f}_{i}$ as follows:

$$
\boldsymbol{f}_{i}(\overrightarrow{\boldsymbol{c}})=D_{i} \boldsymbol{c}_{i}+\boldsymbol{s}_{i} \oslash \boldsymbol{v}+\mathbf{r}_{i}(\overrightarrow{\boldsymbol{c}}), i=1, \ldots, m_{s} .
$$

Equation (2.6) is now written as (cf. (1.2)):

$$
\frac{\partial \boldsymbol{c}_{i}}{\partial t}=F_{i}(\overrightarrow{\boldsymbol{c}}) \equiv\left(T\left(\boldsymbol{c}_{i} \odot \boldsymbol{v}\right)\right) \oslash \boldsymbol{v}+\boldsymbol{f}_{i}(\overrightarrow{\boldsymbol{c}}), i=1, \ldots, m_{s} .
$$

\section{InTEGRATION METHODS}

The chemistry processes are continuously driven by rapidly changing photolysis rates. As a result, chemistry is very stiff and implicit integration is indispensible. A typical time step that is desirable for the other processes is in the order of 30 minutes. This time step enables the simulation of long term changes in the atmospheric composition which is one of the main application areas of the TM3 model. For the time step $\tau=30$ minutes one normally has

$$
\tau\left\|f^{\prime}\right\|_{2} \sim \mathcal{O}\left(10^{6}\right), \quad \tau\|T\|_{2} \sim \mathcal{O}(10),
$$

whereas the smallest in modulus eigenvalues are of order $\mathcal{O}\left(10^{-5}\right)$.

All three methods for solving (1.2) are derived from a particular Rosenbrock method (which we call Ros2) from the stiff ODE field $[6,11]$. Let $\gamma=1+\frac{1}{2} \sqrt{2}$ and denote $A=F^{\prime}\left(w_{n}\right)$. The Rosenbrock method reads

$$
\begin{aligned}
w_{n+1} & =w_{n}+\frac{1}{2} k_{1}+\frac{1}{2} k_{2}, \\
(I-\gamma \tau A) k_{1} & =\tau F\left(w_{n}\right), \\
(I-\gamma \tau A) k_{2} & =\tau F\left(w_{n}+k_{1}\right)-2 \gamma \tau A k_{1},
\end{aligned}
$$


where $w_{n} \approx w\left(t_{n}\right)$ and $\tau=t_{n+1}-t_{n}$ denotes the step size. An equivalent formulation, more attractive for implementation, is

$$
\begin{aligned}
w_{n+1} & =w_{n}+\frac{3}{2} k_{1}+\frac{1}{2} k_{2}, \\
(I-\gamma \tau A) k_{1} & =\tau F\left(w_{n}\right), \\
(I-\gamma \tau A) k_{2} & =\tau F\left(w_{n}+k_{1}\right)-2 k_{1} .
\end{aligned}
$$

With this formulation we economize on a matrix-vector multiplication. This two-stage method is 2nd-order consistent and it has the stability function $R(z)=(1+(1-2 \gamma) z) /(1-\gamma z)^{2}$ which is $L$-stable and positive for all $z \leq 0$. In [3,27] it is argued that this low order, linearly implicit method is suitable for solving stiff atmospheric chemical kinetics problems (box models), provided the costs for the linear system solutions and the Jacobian updates are minimized by using optimal sparsity routines. Such routines are provided by the chemical kinetics preprocessor KPP [4, 5, 23]. Ros2 remains order two consistent with an arbitrary matrix $A, A \neq F^{\prime}\left(w_{n}\right)$. However, stability of the scheme is corrupted when $A$ is not the exact Jacobian.

As is explained in Section 1, straightforward application of method (3.3) to the coupled verticaltransport-chemistry problem is expensive. In full three-dimensional computations the costs for the linear systems solutions with the nearly full and large matrices $F^{\prime}\left(w_{n}\right)=I-\gamma \tau\left(T+f^{\prime}\left(w_{n}\right)\right)$ would be unacceptably large. But solving linear systems with the submatrices $I-\gamma \tau T$ and $I-\gamma f^{\prime}\left(w_{n}\right)$ is economical, as both are block-diagonal (upon reordering, cf. Section 2). This property, with a few others, largely determines the choice for the three integration methods discussed next.

\subsection{Strang splitting}

Let $\Phi_{T}(t ; \tau)$ and $\Phi_{f}(t ; \tau)$ denote the Ros2 integrator (3.3) applied to $\dot{w}=T w$ and $\dot{w}=f(w)$, respectively. The Strang-type operator splitting method for system (1.2) using a stepsize $\tau$ and ending with a chemistry step [24], can then be formulated as

$$
w_{n+1}=\Phi_{f}\left(t_{n+1 / 2} ; \frac{1}{2} \tau\right) \Phi_{T}\left(t_{n+1 / 2} ; \frac{1}{2} \tau\right) \Phi_{T}\left(t_{n} ; \frac{1}{2} \tau\right) \Phi_{f}\left(t_{n} ; \frac{1}{2} \tau\right) w_{n} .
$$

This method is 2nd-order consistent. The leading local error term is the sum of the leading local errors made by the Rosenbrock method and the leading local splitting error. The latter is zero in case $T w$ and $f(w)$ commute $[8,18]$. This, however, is in general not true. In what follows, we refer to method (3.4) as STRANG.

The STRANG method is an improved version of a splitting method used in the TM3 model [22]. The essential difference from (3.4) is that the vertical transport is integrated with larger step sizes of order several hours alternatively with series of still small chemistry substeps. Also, in the splitting method used in TM3 the deposition and source operators are split from the chemistry operator. Since the vertical transport matrix changes only every 6 hours, its inverse is also recomputed only every 6 hours, the same number of times in both methods (3.4) and the splitting method used in TM3. Thus, with a relatively small computational overhead, (3.4) appears an attractive alternative to the splitting method in TM3.

The stability properties of the STRANG method are largely determined by those of each of its substeps: since at each substep the $L$-stable Ros2 method is applied, the sTrang method is $L$-stable.

The splitting in both methods has adverse effects on especially the integration of the stiff chemistry: initial values for the chemistry substeps are not always the results of the previous chemistry substeps, they are 'discontinuous' for the chemistry integration. This leads to so-called stiff transients. The methods considered in the next two sections are aimed to avoid this artifact.

\subsection{S-SPLIT: source splitting method}

In the source splitting method [16, 15], one of the split processes is approximated at each time step as a constant source to be applied with the other split process. In fact, we replace (1.2) with

$$
\dot{w}=\hat{F}(w)=f(w)+S, \quad t \in\left[t_{n}, t_{n}+\tau\right],
$$


where $S$ is the source term approximating vertical transport. The scheme can be written as

$$
\begin{aligned}
\tilde{w}_{n+1} & =\Phi_{T}\left(t_{n} ; \tau\right) w_{n}, \\
S & =\frac{\tilde{w}_{n+1}-w_{n}}{\tau}, \\
w_{n+1} & =\Phi_{\hat{F}}\left(t_{n}, \tau\right) w_{n} .
\end{aligned}
$$

Formally, splitting has been removed because the step $\Phi_{\hat{F}}$ is in fact done for the unsplit system (3.5) that approximates the full system (1.2). Independently of the order of the methods used per substep in (3.6) and (3.7), this S-SPLIT method is only first order consistent. Observe that $S=T w+\mathcal{O}(\tau)$. Just as in standard splitting, in S-SPLIT there is essentially the same freedom left to use one's favorite combination of algorithms. Higher order generalizations of the source splitting are considered in [17].

We note in passing that it is profitable to have the substep corresponding to the stiffest process, chemistry, at the end of the splitting sequence [24]. This is the case for methods STRANG and S-SPLIT.

To get an impression of the stability of the S-SPLIT method, we consider the standard scalar test equation

$$
\dot{w}=\lambda_{f} w+\lambda_{T} w .
$$

Denoting $z_{f}=\tau \lambda_{f}$ and $z_{T}=\tau \lambda_{T}$, we can write the stability function of the S-SPLIT method as

$$
R\left(z_{f}, z_{T}\right)=R_{0}\left(z_{f}\right)+R_{1}\left(z_{f}\right)\left[R_{0}\left(z_{T}\right)-1\right],
$$

where

$$
R_{0}(z)=\frac{1+(1-2 \gamma) z}{(1-\gamma z)^{2}}, \quad R_{1}(z)=\frac{1+\left(\frac{1}{2}-2 \gamma\right) z}{(1-\gamma z)^{2}} .
$$

Here $R_{0}(z)$ is the stability function of the Ros2 method and function $R_{1}(z)$ is such that a Ros2 step for the equation $\dot{w}=\lambda w+\sigma$ can be written as

$$
w_{n+1}=R_{0}(z) w_{n}+R_{1}(z) \tau \sigma .
$$

Simple analysis leads us to the following observations:

(a) Fix $z_{T}=z_{T}^{*} \leq 0$. Then function $R_{f}\left(z_{f}\right)=R\left(z_{f}, z_{T}^{*}\right)$ is $L$-stable,but not positive for $z_{f} \leq 0$.

(b) Fix $z_{f}=z_{f}^{*} \leq 0$. Then function $R_{T}\left(z_{T}\right)=R\left(z_{f}^{*}, z_{T}\right)$ is $A$-stable, but not positive for $z_{T} \leq 0$.

Property (a) quarantees good damping for large chemistry eigenvalues $\lambda_{f}$, whereas the lack of $L$-stability in (b) is hardly harmful taking into account that vertical transport eigenvalues are normally several orders less than those of chemistry. Positivity of $R$ is argued to be important for chemistry kinetic problems in [27], so, for S-SPLIT lacking this property, we can expect positivity problems. Indeed, in our numerical experiments we have observed that negative values in chemistry computations were encountered in the S-SPLIT scheme sligthly more often than in STRANG (unphysical negative concentrations occuring occasionally in computations were set to zero).

\subsection{ROS2-AMF: approximate factorization}

The Ros2-AMF method is the Ros2 scheme (3.3) applied to the whole system (1.2) with an inexact matrix $I-\gamma \tau A$. For $I-\gamma \tau A$ we take Approximate Matrix Factorization (AMF) in the form:

$$
I-\gamma \tau A=(I-\gamma \tau T)\left(I-\gamma \tau f^{\prime}\right) .
$$

This Ros2-AMF method was introduced in [27] and recently tested in [3]. The idea of approximate matrix factorization is known at least since papers [2, 9, 28] and still widely used (see e.g. [1, 14]).

The Ros2-AMF method is split-free because the 'splitting' appears only at the level of the linear solves with matrix $I-\gamma \tau A$. However, the method has retained the ease of implementation of the standard splitting: the algorithmic ingredients (among which the linear solves with matrices $I-\gamma \tau T$ and $I-\gamma \tau f^{\prime}$ are the most essential) remain basically the same as in conventional splitting. Taking an inexact Jacobian in (3.8) leads to only very moderate loss of stability: in fact, the scheme maintains its $A$-stability, only the $L$-stability is lost [27]. 


\subsection{Computational costs}

Methods S-SPLIT and ROS2-AMF require the same work per time step and, since the whole step of STRANG consists of two substeps for each process (cf. (3.4)), two steps of either of the methods are equal in cost to one step of STRANG. In our experiments we use the same constant step size $\tau$ for S-SPLIT and ROS2-AMF and twice as large a step size for STRANG. This makes these three methods equal in costs.

The work per step of S-SPLIT or ROS2-AMF consists essentially of one evaluation of the chemistry Jacobian $f^{\prime}$, one sparse LU factorization of $I-\gamma \tau f^{\prime}$, two linear system solves with each of the matrices $I-\gamma \tau f^{\prime}$ and $I-\gamma \tau T$, two chemistry function evaluations and two matrix-vector multiplication with the vertical transport matrix $T$. Matrices $T$ and $(I-\gamma \tau T)^{-1}$ are updated every 6 hours.

\section{Numerical COMPARISONS AND DISCUSSION}

We have run the S-SPLIT and ROS2-AMF methods with a step size $\tau=30 \mathrm{~min}$ and the STRANG method with a step size $\tau=60 \mathrm{~min}$. With these step sizes all three methods are equal in computational costs (see Section 3.4). These step sizes are quite large for the chemistry reaction set used (cf. (3.1)).

We compare the accuracy of the methods against a reference solution. The reference solution was obtained by the STRANG method run with a tiny step size $\tau=3 \mathrm{sec}$. With such a step size, all three methods converged to the same solution.

Despite the large time steps chosen, all three methods perform well delivering notably small errors (mostly less than $10 \%$ of the daily variations). Since all the methods are based on the Ros2 time stepping scheme, one may conclude that this integrator is stable and accurate.

In Figure 1 we plot the error $\epsilon_{k ; n}$ as a function of time level $n$ and of height $k$ for the method ROS2-AMF. In Figure 2, we plot the height-average of the error $\epsilon_{k ; n}$ of the three methods as functions of local time. In these figures the local-time interval ranges from 9 to 12 March. In Figure 3, we plot the time-average of the error $\epsilon_{k ; n}$ (for 6 to 15 March) of the three methods as functions of the vertical level. The error $\epsilon_{k ; n}$ in cell $k$ and at time $n$ is defined as the scaled distance in the $m_{s}$-dimensional space of the concentration vectors $\vec{c}_{k ; n}$ of a particular method and $\vec{c}_{k ; n}^{\text {ref }}$ of the reference solution. The formal definition is:

$$
\epsilon_{k ; n} \equiv\left\|\left(\vec{c}_{k ; n}-\vec{c}_{k ; n}^{\mathrm{ref}}\right) \oslash\left(\max _{k ; n} \vec{c}_{k ; n}^{\mathrm{ref}}-\min _{k ; n} \vec{c}_{k ; n}^{\mathrm{ref}}\right)\right\|_{2} / \sqrt{m_{s}},
$$

where the max and min operators work elementwise, i.e. per species. The distances are scaled elementwise with the total range of the concentration in the reference solution. They are also scaled with $\sqrt{m_{s}}$. This guarantees that $\epsilon_{k ; n}$ lies between the smallest and the largest relative error per species.

As we see in Figure 1, ROS2-AMF produces larger errors in the morning and in the evening. Since the chemistry is driven by photolysis rates that change rapidly with the solar zenith angle, the reference solution (time step of 3 seconds) is able to capture this rapid change better than the other solutions, which use much larger time steps. The errors also become larger at the times when the vertical transport update occurs during daytime, notably at $8 \mathrm{~h}$ and $14 \mathrm{~h}$ local time. However, the errors of all the methods remain small and do not grow in time. Quite similar behaviour also occurs for S-SPLIT and STRANG (not shown). In [3], Figures 6 and 7, it is also seen that large errors in the solution for the short-lived species $\mathrm{N}_{2} \mathrm{O}_{5}$ mainly occur — or start and end - at sunrise and sunset.

In general Ros2-AMF appears more accurate than both STRANG and S-SPLIT, and S-SPLIT is slightly more accurate than STRANG. However, this is not generally true for every tracer in each layer. However, it can be observed in Figure 3 that ROS2-AMF performs better than both STRANG and S-SPLIT in most of the layers. Since ROS2-AMF does not split the emission and deposition processes to the vertical transport, these differences are due to split errors that occur in S-SPLIT and STRAng. This observation is emphasized in Figures 4 to 8, which show the concentration as a function of time for a few selected tracers in the lowest model layer. Their lifetimes range from 


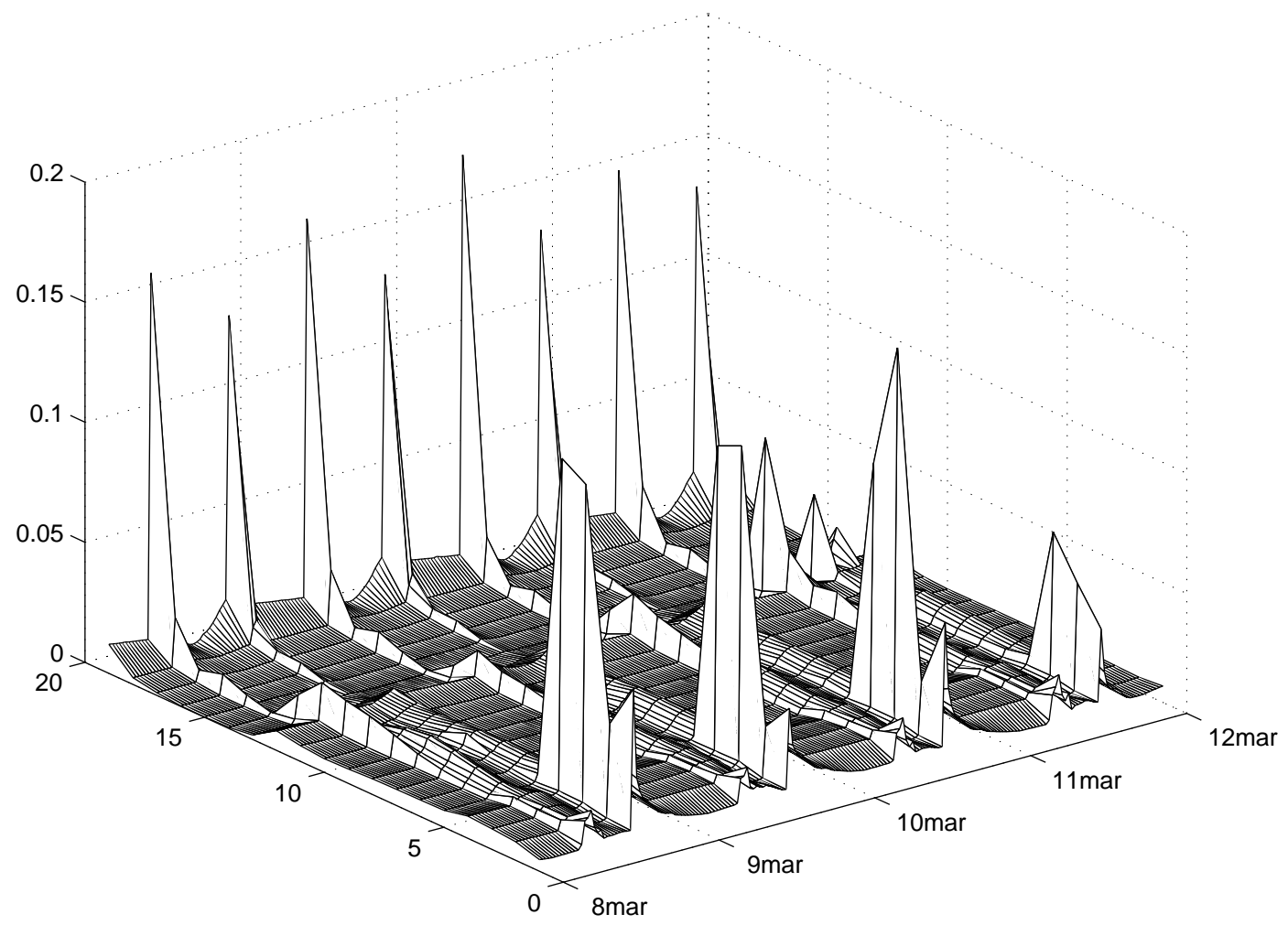

Figure 1: The error $\epsilon_{k ; n}$ for the method Ros2-AMF as a function of time and model level.

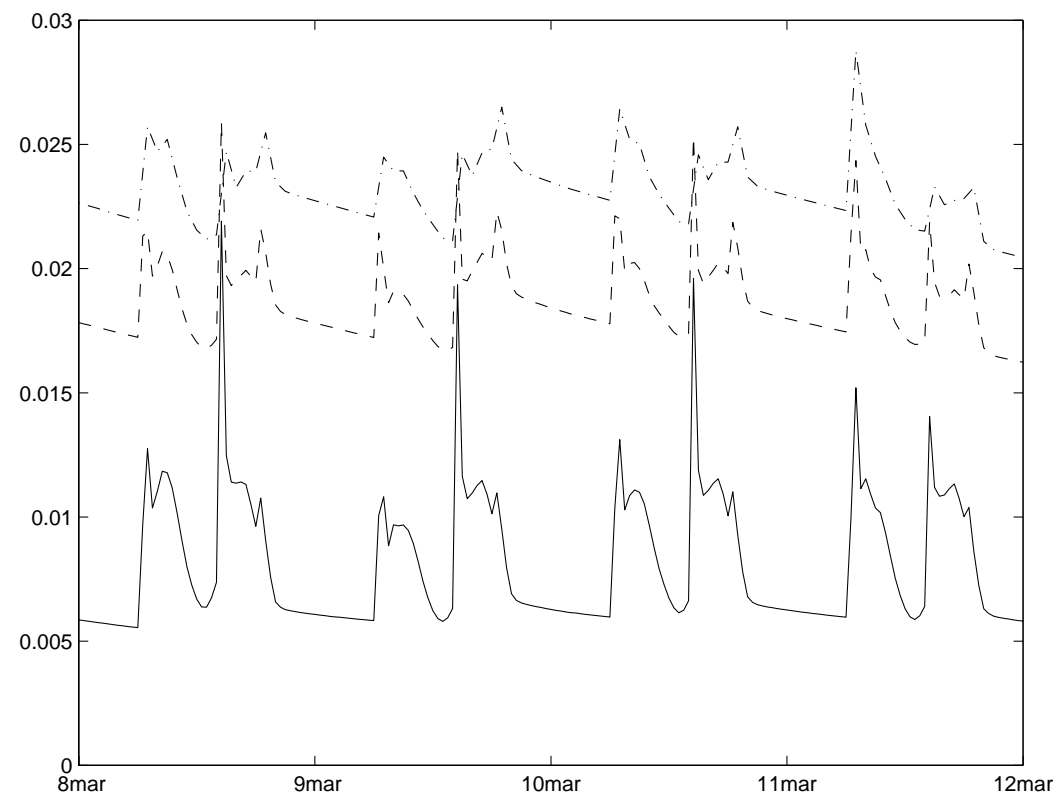

Figure 2: The height-averaged error as a function of time (solid line for Ros2-AMF, dashed line for S-SPLIT, and dash-dotted line for STRANG). 
seconds $(\mathrm{OH})$ to several hours $\left(\mathrm{O}_{3}\right)$. Due to reduced splitting errors, ROS2-AMF performs best compared to the reference solution.

For each method we have also calculated the time and layer averaged relative contributions per species to the total error defined in (4.1). The results for STRANG and S-SPLIT are almost identical, with very large contributions for $\mathrm{NH}_{3}$ and $\mathrm{HNO}_{4}$. For ROS2-AMF they differ considerably in that the errors are more evenly distributed over the species. For all methods, the species that contribute most to the total error are those species which react strongly to changes in solar radiation (the only exception being $\mathrm{NH}_{3}$ ). This happens at sunrise and sunset, as well as at the times of the vertical transport updates, when the cloudiness changes also. Occasionally these errors are larger than $10 \%$ of the daily variations. This confirms the finding in [3] that $\mathrm{N}_{2} \mathrm{O}_{5}$ has large errors, which are related to sunrise and sunset. As mentioned, the only exception is $\mathrm{NH}_{3}$, which is not (photo)chemically active in this model. However, it has strong emissions and (solar radiation dependent) depositions, which are split from the vertical transport in the numerical methods, see the argumentation and equation (2.7) in Section 2.2.

In general, both S-SPLIT and STRANG, based on the standard operator splitting, perform well. However, it seems to be crucial for the performance of STRANG that the step size for the vertical substep is not taken larger than for the chemistry substep. Our experiments showed that alternating two 3-hour vertical-transport substeps with three 2-hour groups of half an hour chemistry substeps within a 6-hour timestep (i.e. the splitting method used in TM3 with a 6-hour time step) leads to dramatical loss of accuracy. Errors may reach the order of magnitude of the daily concentration variations. On the other hand, STRANG seems to be not very dependent on whether emissions and depositions are included in the chemistry substep or handled separately as an extra operator in the splitting. We note that S-SPLIT, though being a first order method, compares well with the second order STRANG and ROS2-AMF. Again, it is likely that this is caused by reduced splitting in the S-SPLIT method.

As noted earlier, the time accurate reference solution accurately follows the rapid transitions in the morning and evening period. The error calculated for the ROS2-AMF method might therefore be due to the non-autonomous nature of the problem. In order to investigate this, we compared the ROS2-AMF scheme with Ros2 applied to the whole unsplit problem with a time step of 30 minutes. We already noted that this scheme is by far more expensive than the other schemes. The solution produced by this fully implicit Ros2 scheme was hardly distinguishable from the cheap ROS2-AMF solution. This indicates that, at least in this particular case, the AMF provides a good approximation to the whole Jacobian linear system (cf. (3.8)).

\section{Conclusions}

In a column submodel of the global dispersion model TM3, with strong mixing and stiff chemistry, we have compared the standard operator splitting method STRANG versus two other methods S-SPLIT and ROS2-AMF with (partially) eliminated splitting.

Our conclusions are:

- With relatively large time steps (of half an hour), all three methods perform well. Generally the errors in the concentrations are only a few percent of the total daily variations.

- Such accuracy is by far sufficient for the application area. An error limit of $10 \%$ is acceptable in atmospheric chemistry modelling, since other error sources, e.g. emissions, cause errors of similar or larger magnitude.

- In general the ROS2-AMF scheme appears to be the most accurate scheme, giving the smallest errors for most tracers and layers. In particular this is the case for the important surface layer where ROS2-AMF clearly gives the best solution.

- Since the only differences between the three methods are in the degree and the way of splitting, the differences in the errors must be caused by differences in operator splitting. In the present experiment this implies that splitting errors result from splitting chemistry (including emission and deposition) and transport. The higher accuracy of Ros2-AMF as 


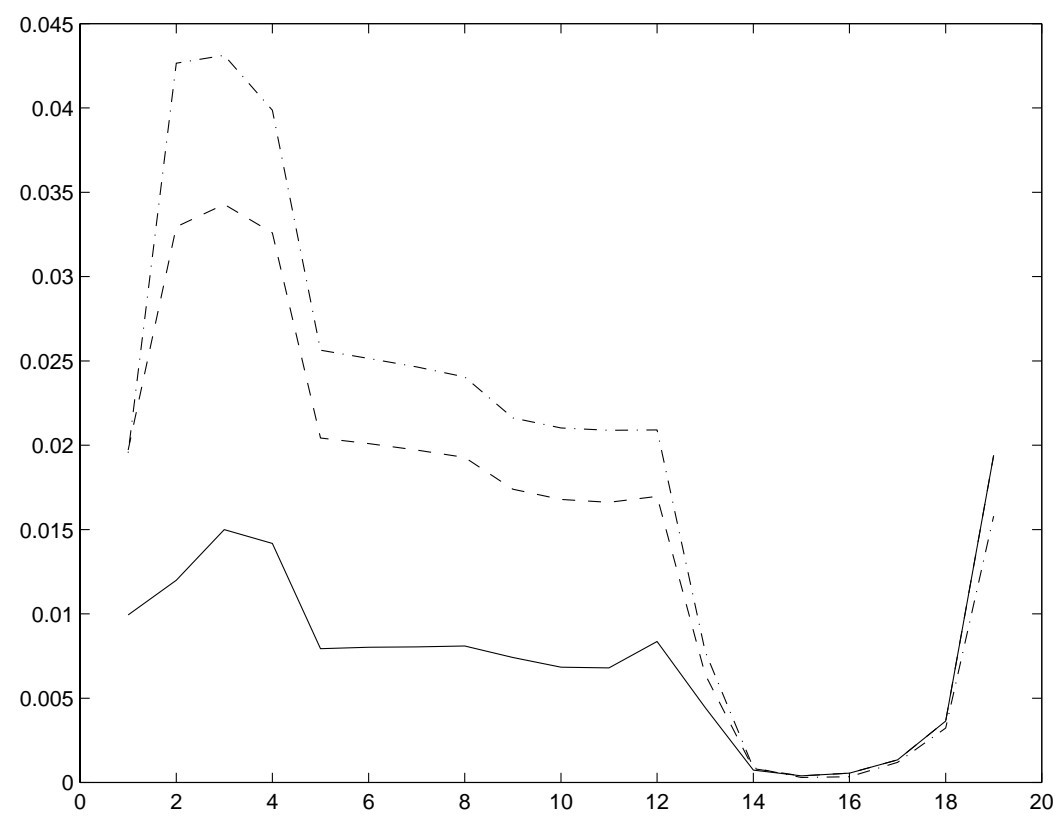

Figure 3: The time-averaged error as a function of model level (solid line for Ros2-AMF, dashed line for S-SPLIT, and dash-dotted line for STRANG).
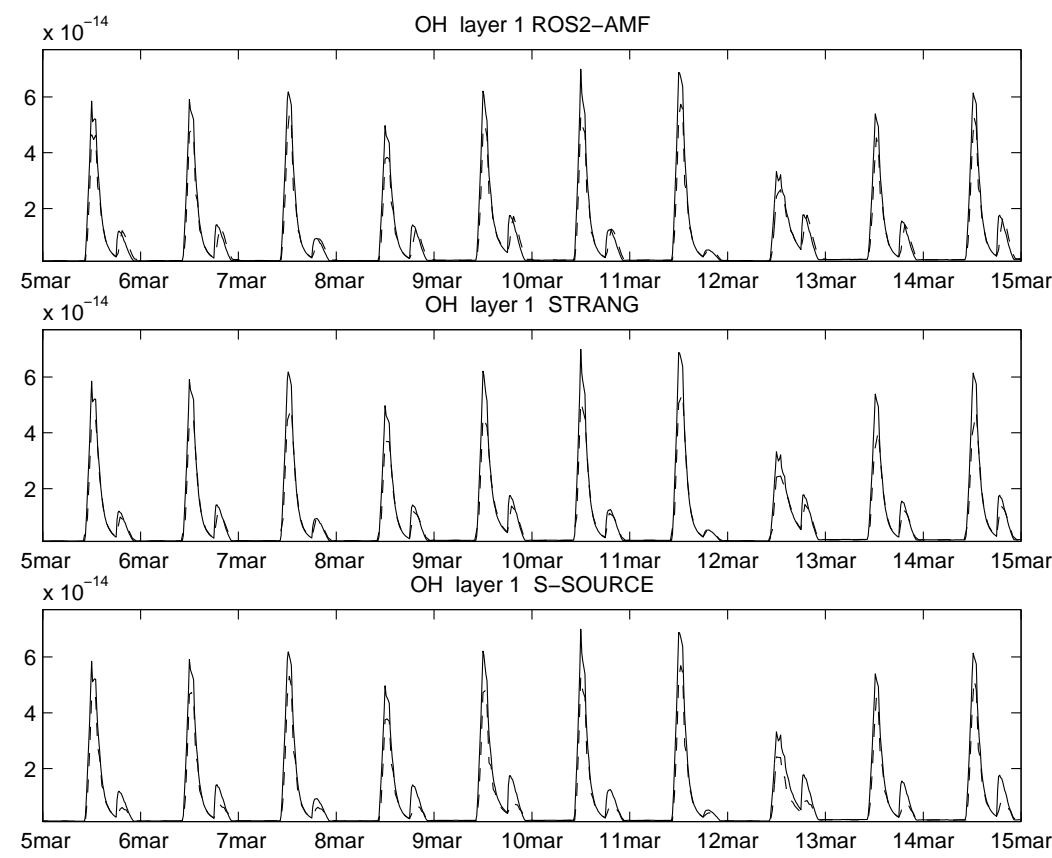

Figure 4: The $\mathrm{OH}$ solution in the surface cell as a function of time in molecules per air molecules (volume mixing ratio). 

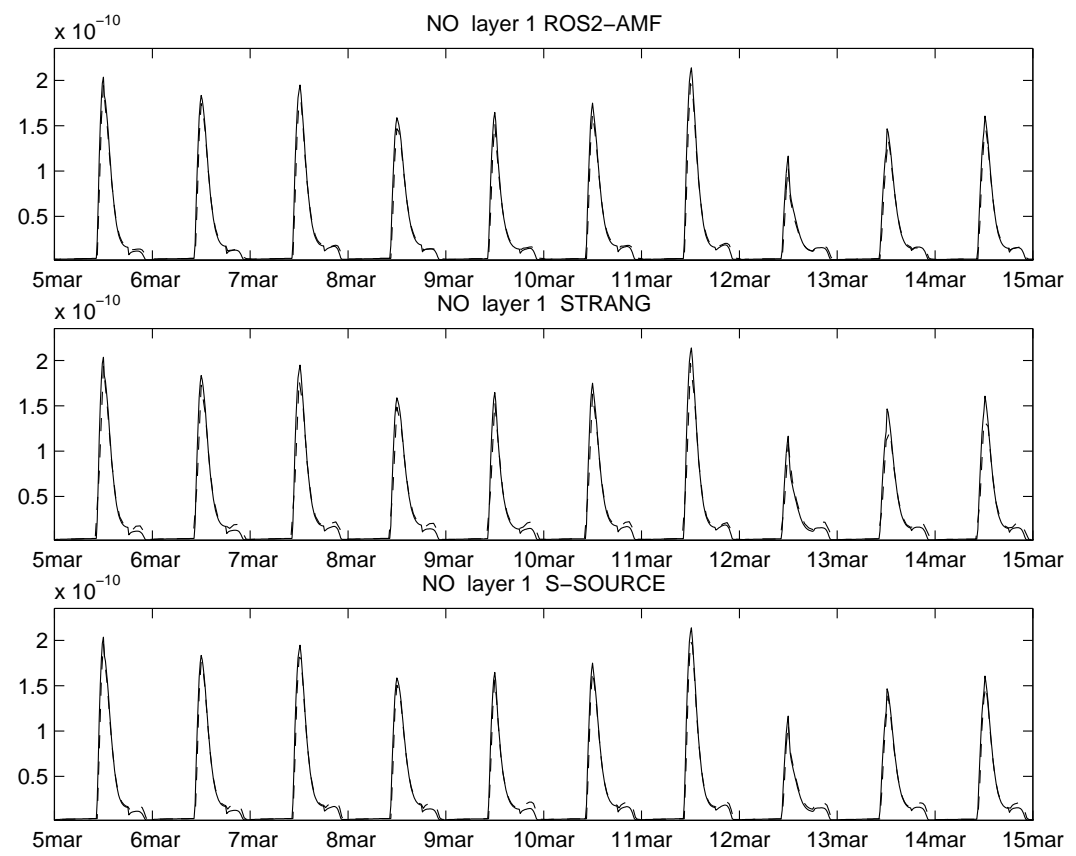

Figure 5: The NO solution in the surface cell as a function of time in molecules per air molecules (volume mixing ratio).
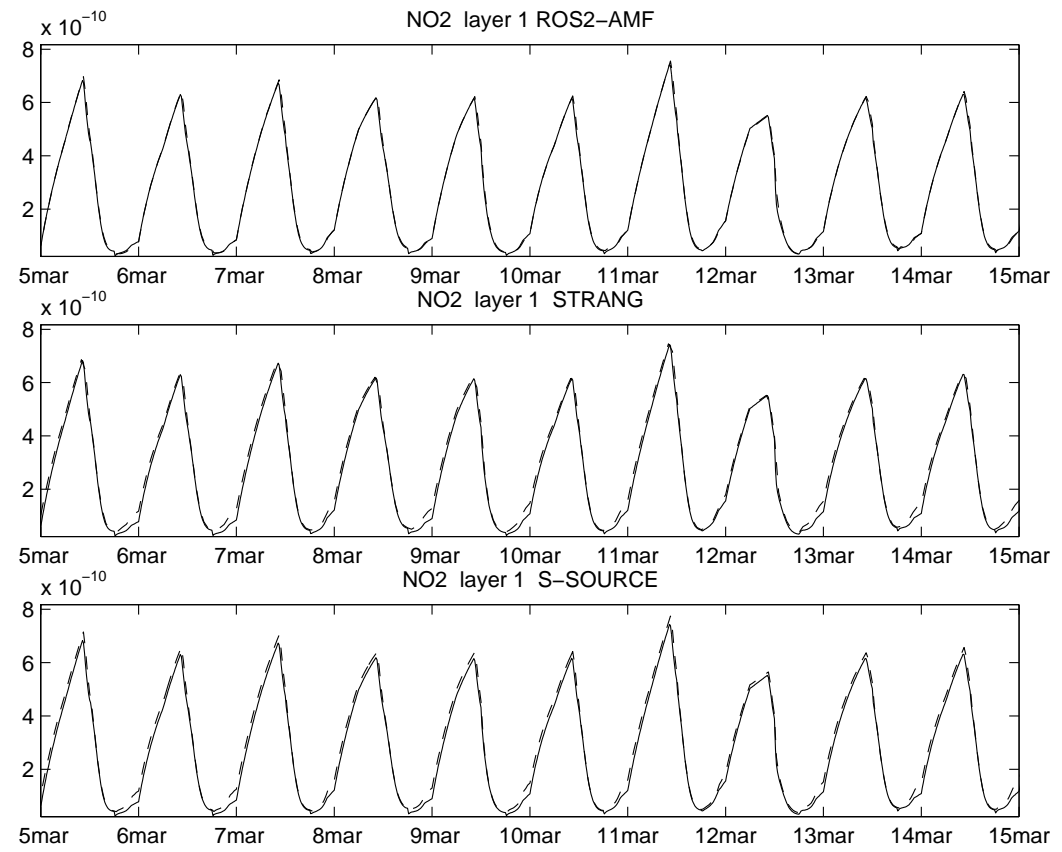

Figure 6: The $\mathrm{NO}_{2}$ solution in the surface cell as a function of time in molecules per air molecules (volume mixing ratio). 

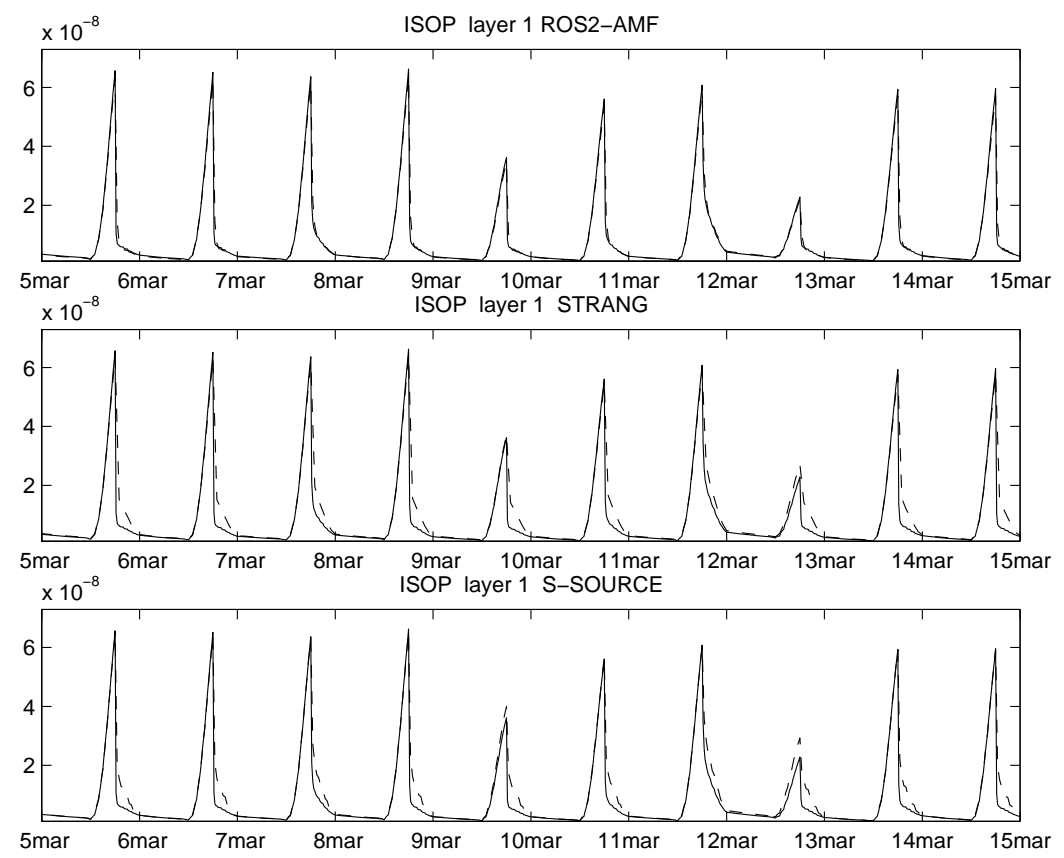

Figure 7: The ISOP solution in the surface cell as a function of time in molecules per air molecules (volume mixing ratio).
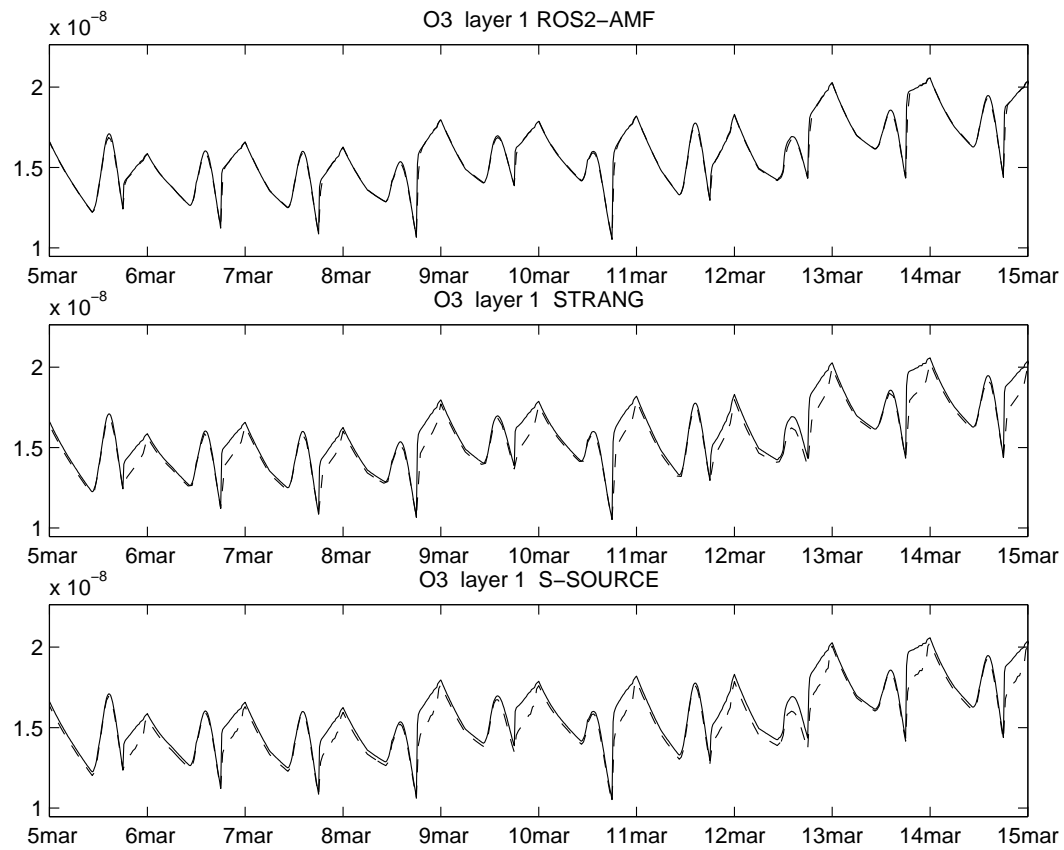

Figure 8: The $\mathrm{O}_{3}$ solution in the surface cell as a function of time in molecules per air molecules (volume mixing ratio). 
compared to STRANG and S-SPLIT is therefore attributed to the absence of operator splitting in the former method. We note that the first-order S-SPLIT compares favorably with the second-order STRANG. This is attributed to the fact that splitting has been formally removed in S-SPLIT.

Unacceptably large splitting errors remain restricted to short-lived species sensitive to solar radiation and those with strong emissions and depositions.

For the accuracy of a standard operator splitting method (notably the splitting method used in TM3) we found that it is crucial not to interchange the relatively small substeps of the stiff process (chemistry) with much larger timesteps for the not-so-stiff process (transport), even if stability allows this.

- The computational costs of all three methods are essentially the same. Approximate Matrix Factorization (AMF) allows to apply the Ros2 scheme to the whole unsplit problem in an efficient way, thus avoiding splitting at no extra cost.

- Since all the schemes are based on the Ros2 integrator, we conclude that Ros2 proves successful for this difficult problem, and, based also on conclusions in [3], we recommend Ros2 as a viable method for use in the field of air pollution modelling.

Acknowledgements This work has been sponsored by the Netherlands Organization for Scientific Research (NWO) under grants no. 613-302-040, 750-298-02, by the Space Research Organization Netherlands (SRON), and by the Netherlands Institute for Computer Facilities (NCF). 


\section{References}

1. I. Ahmad and M. Berzins. An algorithm for ODEs from atmospheric dispersion problems. Appl. Num. Math., 25:137-150, 1997.

2. R. M. Beam and R. F. Warming. An implicit finite-difference algorithm for hyperbolic systems in conservation-law form. J. Comp. Phys., 22:87-110, 1976.

3. J. G. Blom and J. G. Verwer. A comparison of integration methods for atmospheric transportchemistry methods. J. Comp. Appl. Math., to appear in 2001.

4. V. Damian-Iordache. KPP - chemistry simulation development environment. Master's thesis, University of Iowa, 1996.

5. http://www.cgrer.uiowa.edu/people/vdamian/kpp/.

6. K. Dekker and J. G. Verwer. Stability of Runge-Kutta methods for stiff non-linear differential equations. North-Holland Elsevier Science Publishers, 1984.

7. F. J. Dentener, J. Feichter, and A. Jeuken. Simulation of transport of ${ }^{222} \mathrm{Rn}$ using on-line and off-line global models at different horizontal resolutions: A detailed comparison with measurements. Tel. Ser. B, 51:573-602, 1999.

8. I. Dimov, I. Faragó, and Z. Zlatev. Commutativity of the operators in splitting methods for air pollution models. Technical Report 04/99, Central Laboratory for Parallel Processing, Bulgarian Academy of Sciences, Sofia, 1999.

9. E. G. D'yakonov. Difference systems of second order accuracy with a divided operator for parabolic equations without mixed derivatives. USSR Comput. Math. Math. Phys., 4(5):206216, 1964.

10. W. Guelle, Y. J. Balkanski, M. Schulz, F. Dulac, and P. Monfray. Wet deposition in a global size-dependent aerosol transport model, 1 , comparison of a 1 year ${ }^{210} \mathrm{~Pb}$ simulation with ground measurements. J. Geophys. Res., 103:11,429-11,445, 1998.

11. E. Hairer and G. Wanner. Solving Ordinary Differential Equations II. Stiff and DifferentialAlgebraic Problems. Springer-Verlag, Berlin, 2nd edition, 1996.

12. M. Heimann. The global atmospheric tracer model TM2. Technical report, DKRZ (Deutsches Klimarechenzentrum), Hamburg, Germany, 1995.

13. S. Houweling, F. J. Dentener, and J. Lelieveld. The impact of non-methane hydrocarbon compounds on tropospheric photochemistry. J. Geophys. Res., 103:10,673-10,696, 1998.

14. P. J. v. d. Houwen and B. P. Sommeijer. Approximate factorization for time-dependent partial 
differential equations. J. Comp. Appl. Math., 2000. Report MAS-R9915, CWI (Centre for Mathematics and Computer Science), Amsterdam, The Netherlands.

15. C. Kessler. Entwicklung eines effizienten Lösungsverfahrens zur modellmäßigen Beschreibung der Ausbreitung und chemischen Umwandlung reaktiver Luftschadstoffe. PhD thesis, Fakultät für Maschinenbau der Universtät Karlsruhe, 1995.

16. O. Knoth and R. Wolke. A comparison of fast chemical kinetic solvers in a simple vertical diffusion model. In S.-V. Gryning and M. M. Millán, editors, Air Pollution Modelling and Its Application, volume X, pages 287-294. Plenum Press, 1994.

17. O. Knoth and R. Wolke. Implicit-explicit Runge-Kutta methods for computing atmosperic reactive flows. Appl. Num. Math., 28:327-341, 1998.

18. D. Lanser and J. G. Verwer. Analysis of operator splitting for advection-diffusion-reaction problems from air pollution modelling. J. Comp. Appl. Math., 111:201-206, 1999.

19. J. Lelieveld and F. J. Dentener. What controls tropospheric ozone. J. Geophys. Res., 105:3531, 2000.

20. J. F. Louis. A parametric model of vertical eddy fluxes in the atmosphere. Bound. Layer Met., 17:178-202, 1979.

21. G. J. McRae, W. R. Goodin, and J. H. Seinfeld. Numerical solution of the atmospheric diffusion equation for chemically reacting flows. J. Comp. Phys., 45:1-42, 1982.

22. http://www.phys.uu.nl/ peters/TM3/TM3S.html.

23. A. Sandu, F. A. Potra, G. R. Carmichael, and V. Damian. Efficient implementation of fully implicit methods for atmospheric chemical kinetics. J. Comp. Phys., 129:101-110, 1996.

24. B. Sportisse. An analysis of operator splitting techniques in the stiff case. Technical Report 98-127, ENPC-CERMICS, Champs sur Marne, France, 1998.

25. M. Tiedtke. A comprehensive mass-flux scheme for cumulus parameterization in large-scale models. Mon. Weather Rev., 117:1779-1800, 1989.

26. J. G. Verwer, W. Hundsdorfer, and J. G. Blom. Numerical time integration for air pollution models. Surv. Math. Ind., to appear in 2001. Report MAS-R9825, CWI (Centre for Mathematics and Computer Science), Amsterdam, The Netherlands.

27. J. G. Verwer, E. J. Spee, J. G. Blom, and W. Hundsdorfer. A second order Rosenbrock method applied to photochemical dispersion problems. SIAM J. Sci. Comp., 20:1456-1480, 1999.

28. R. F. Warming and R. M. Beam. An extension of A-stability to alternating direction methods. BIT, 19:395-417, 1979.

29. M. L. Wesely. Parameterization of surface resistance to gaseous dry deposition in regional-scale numerical models. Atm. Env., 23:1293-1304, 1989.

30. Z. Zlatev. Computer Treatment of Large Air Pollution Models. Kluwer Academic Publishers, 1995. 